CLINICAL STUDY

\title{
Increased progression of carotid intima media thickness in thyroid peroxidase antibodies-positive rheumatoid arthritis patients
}

\author{
H G Raterman ${ }^{1}$, A E Voskuyl ${ }^{1}$, S Simsek ${ }^{2,3}$, M W J Schreurs ${ }^{4,5}$, I M W van Hoogstraten ${ }^{4}$, M J L Peters ${ }^{2}$, \\ V P van Halm ${ }^{1,6}$, B A C Dijkmans ${ }^{1,7}$, P Lips ${ }^{2}$, W F Lems ${ }^{1,7}$ and M T Nurmohamed ${ }^{1,2,7}$ \\ Departments of ${ }^{1}$ Rheumatology and ${ }^{2}$ Internal Medicine, VU University Medical Center, Postbus 7057, 1007 MB Amsterdam, The Netherlands, \\ ${ }^{3}$ Department of Internal Medicine, Medical Centre Alkmaar, Postbus 501, 1800 AM Alkmaar, The Netherlands, ${ }^{4}$ Department of Pathology, VU University \\ Medical Center, Postbus 7057, 1007 MB Amsterdam, The Netherlands, ${ }^{5}$ Department of Immunology, Erasmus MC, University Medical Center, \\ Dr Molewaterplein 50, 3015 GE Rotterdam, The Netherlands, ${ }^{6}$ Department of Cardiology, Academical Medical Center, Meibergdreef 9, 1105 AZ \\ Amsterdam, The Netherlands and ${ }^{7}$ Department of Rheumatology, Jan van Breemen Research Institute/Reade, Postbus 582711040 HG Amsterdam, \\ The Netherlands
}

(Correspondence should be addressed to H G Raterman; Email: h.raterman@vumc.nl)

\begin{abstract}
Objective: Autoimmune diseases such as rheumatoid arthritis (RA) and hypothyroidism tend to cluster, and this coexistence amplifies the elevated cardiovascular risk in RA. Whether thyroid peroxidase antibodies (TPOabs) are associated with increased cardiovascular disease (CVD) risk has not been studied extensively. Therefore, this study determined firstly the prevalence of TPOabs in RA and secondly whether TPOabs were associated with CVD. Moreover, this study explored whether TPOabs were related to RA characteristics.

Design and methods: Data from the CARRÉ Study, an ongoing study investigating CVDs and its risk factors in RA $(n=322)$, was used to ascertain the prevalence of TPOabs in RA patients. In addition, cardiovascular and RA disease characteristics were compared between TPOabs-positive and -negative patients at baseline and at a second visit after 3 years.

Results: TPOabs were present in 47/322 (15\%) RA patients and TSH levels were higher in TPOabspositive patients $(1.40 \mathrm{mU} / \mathrm{l})$ compared with TPOabs-negative patients $(1.26 \mathrm{mU} / \mathrm{l}, \mathrm{P}=0.048)$. At baseline and after 3 years no association was observed between TPOabs and (risk factors for) CVD. Regression analyses revealed a significantly larger progression of carotid intima media thickness (cIMT; $\beta=0.13 \mathrm{~mm}$ ) in TPOabs-positive compared with TPOabs-negative patients independent of risk factors for cIMT progression. RA disease activity scores (DAS28) were higher in TPOabs-positive compared with TPOabs-negative patients (4.4 vs $3.8 \mathrm{P}=0.018)$.

Conclusions: TPOabs were associated with increased cIMT progression. Moreover, an association between TPOabs and DAS28 was observed. Hence, TPOabs seems to have a role in the amplified cardiovascular risk in RA patients.
\end{abstract}

European Journal of Endocrinology 169 751-757

\section{Introduction}

Autoimmune thyroiditis is the most prevalent autoimmune disorder and is characterized by elevated levels of anti-thyroid antibodies in $\sim 70 \%$ of the patients (1). Traditionally, autoimmune diseases such as rheumatoid arthritis (RA) and autoimmune thyroiditis were considered separate disorders, but there is emerging evidence that autoimmune diseases share similarities in genetic and immunological origin, and environmental risk factors $(2,3)$, suggesting that people with one autoimmune disease are at higher risk for another autoimmune disease (4). Coexistence of autoimmune disorders was recently demonstrated for hypothyroidism and RA and, interestingly, this coexistence amplifies the cardiovascular risk $(5,6,7,8,9)$.
The natural course from preclinical to clinical autoimmune diseases such as hypothyroidism and RA is a slow and multifactorial process (10). Therefore, the evolution from pre- or subclinical disease into clinically overt disease takes years. In RA, the presence of anti-cyclic citrullinated protein antibodies (ACPA) is considered an important predictive factor, as ACPA have already been detected a decade before the onset of RA (11). Moreover, ACPA is an important prognostic factor as ACPA is associated with erosive disease and higher mortality rates $(12,13)$. Hence, the autoantibody ACPA has clinical value as a predictor for RA and is also an important prognostic factor in RA patients.

Thyroid peroxidase antibodies (TPOabs) have been implicated as important predictive parameters for future autoimmune hypothyroidism $(14,15,16)$, but on the 
other hand the presence of TPOabs could not be related to prognostic and clinical factors in euthyroid and subclinical hypothyroid patients (17).

Whether the presence of TPOabs (irrespective of thyroid status) is associated with a certain phenotype of RA has not been studied extensively in RA (18). Therefore, the aim of this study was firstly to determine the prevalence of TPOabs in RA and second to determine whether their presence was associated with cardiovascular disease (CVD) or specific parameters of RA.

\section{Subjects and methods}

\section{Study population}

For this study, we used data from the CARRÉ Study, which is a prospective cohort of patients with RA, in whom cardiovascular events and concurrent risk factors were investigated (19). Briefly, in the CARRÉ investigation a total of 353 patients aged $50-75$ years who fulfilled the American College of Rheumatology criteria of 1987 for RA were enrolled (20). Patients were enrolled between 2001 and 2002, and were seen for a second visit between 2004 and 2005 to assess the incidence of risk factors for CVD. This study included RA patients in whom TPOabs could be assessed from baseline samples stored at $-80{ }^{\circ} \mathrm{C}(n=322)$. At followup of the second visit, data of 256 RA patients were used for the analysis. All patients provided written informed consent and the Jan van Breemen Research Institute/Reade received approval by the local medical ethics committee.

\section{Rheumatoid parameters}

All patients with RA attended the outpatient clinic at the Jan van Breemen Research Institute/Reade. These patients were seen by a research physician, and completed a questionnaire recording demographic data, medical, and medication history. A physical examination was performed, including the 28 joint disease activity index score (DAS28) (21). Briefly, DAS28 is a validated instrument to assess the disease activity in RA patients and is a composite of four elements: i) erythrocyte sedimentation rate (ESR); ii) patient's general health or global disease activity on a visual analog scale (VAS) of $100 \mathrm{~mm}$, lower VAS representing better general health; iii) number of swollen joints; and iv) number of painful joints. Furthermore, blood samples were taken to measure the inflammatory and disease-specific parameters: C-reactive protein (CRP) and serological markers (IgM-rheumatoid factor (IgM-RF) and anti-cyclic citrullinated protein antibodies (ACPA)) as previously described (19).

\section{Cardiovascular parameters}

Blood pressure, BMI, and waist-to-hip ratio were assessed as previously described (19). During the visit at the outpatient clinic patients' smoking status was classified as never, current, or former smoker by the research physician. Fasting blood samples were taken to assess lipids and glucose levels. The atherogenic index is defined as the total cholesterol:HDL-C ratio. Diabetes was defined as known diabetes mellitus or patients using at least one glucose-lowering agent.

Metabolic syndrome was defined according to the original National Cholesterol Education Program - Third Adult Treatment Panel (NCEPATP III) definition (22). According to this definition patients fulfil the criteria for metabolic syndrome when three or more of the following factors are present:

1. Abdominal obesity: in females waist circumference $>88 \mathrm{~cm}$ and in males waist circumference $>102 \mathrm{~cm}$;

2. Raised blood pressure: systolic blood pressure $\geq 130 \mathrm{mmHg}$ or diastolic blood pressure $\geq 85 \mathrm{mmHg}$, or use of antihypertensive medication;

3. Raised TG: $\geq 1.7 \mathrm{mmol} / \mathrm{l}(150 \mathrm{mg} / \mathrm{dl})$ or use of lipidlowering drugs;

4. Reduced HDL-C: in females $<1.29 \mathrm{mmol} / \mathrm{l}(50 \mathrm{mg} / \mathrm{dl})$ and in males $<1.03 \mathrm{mmol} / \mathrm{l}(40 \mathrm{mg} / \mathrm{dl})$ or use of lipidlowering drugs;

5. Raised fasting plasma glucose: $\geq 6.1 \mathrm{mmol} / \mathrm{l}$ $(110 \mathrm{mg} / \mathrm{dl})$.

CVDs were defined as a verified history of coronary, cerebral, or peripheral arterial diseases. Coronary artery disease included a myocardial infarction, a coronary artery bypass graft procedure, or percutaneous transluminal coronary angioplasty. Cerebral arterial disease was defined as a cerebral vascular accident, a transient ischemic attack, or carotid endarterectomy. Peripheral arterial disease included a peripheral arterial bypass, an ankle/ brachial blood pressure index of $<0.90$, or leg amputation as a consequence of peripheral arterial disease.

\section{Ultrasonography of the common carotid artery}

In this study, the ultrasound analysis of the right common carotid artery was performed by two physicians, who were unaware of the participants' clinical or laboratory characteristics, as previously described (23). Each observer performed a reproducibility test with other experienced observers from the institute before starting to perform measurements. Inter-observer and intra-observer variability were good (variations $<10 \%$ ). Measurements were performed with a $7.5-\mathrm{MHz}$ linear probe connected to a computer equipped with vessel wall movementdetection software and an acquisition system (Wall track system, Pie Medical) that enables measurement of the carotid intima media thickness (cIMT). After localization of the common carotid artery, cross-sectional 
measurements were performed $10 \mathrm{~mm}$ proximal to the carotid bulb. Sites with mural atherosclerotic plaques were avoided because of difficulty in identifying carotid arterial variables in these regions. The distance between the lumen-intima interface and the leading edge of the media-adventitia interface of the far wall corresponds with cIMT. Measurements of IMT were triggered by echocardiogram to the R-peak of the cardiac cycle. The cIMT was performed in a subset of 105 patients at baseline and in 78 patients after 3 years.

\section{Thyroid parameters}

Thyroid status was assessed at baseline samples. Clinical hypothyroidism was defined by a documented medical history of clinical hypothyroidism or the presence of an increased serum TSH $(>4.0 \mathrm{mU} / \mathrm{l})$ in the presence of a decreased $(<11 \mathrm{pmol} / \mathrm{l})$ serum-free thyroxine $\left(\mathrm{fT}_{4}\right)$, as described previously (5). Subclinical hypothyroidism was defined by an increased serum TSH in the presence of a normal (11-25 pmol/l) serum $\mathrm{fT}_{4}$ and subclinical hypothyroid patients were not treated with levothyroxine (5). TPOabs were determined by immunoassay, using an Immulite 1000 (Siemens, Los Angeles, CA, USA) according to the manufacturer's recommendations. According to these recommendations, the concentration of TPOabs is expressed in $\mathrm{IU} / \mathrm{ml}$ and is considered elevated when levels are higher than $35 \mathrm{IU} / \mathrm{ml}$.

\section{Statistical analyses}

TPOabs prevalence rates were calculated in the CARRE population. Characteristics were compared using Students' $t$-test, Mann-Whitney $U$ test, or $\chi^{2}$ test when appropriate between TPOabs-negative (TPOabs $<35 \mathrm{IU} / \mathrm{ml}$ ) and -positive RA patients. Data were presented as mean ( \pm s.D. $(=$ S.D. $)$ ) in case of a normally distributed parameter or as median with interquartile range in case of a nonnormally distributed parameter, or as percentage of the total population. Linear regression analyses were carried out with difference in cIMT as outcome measure and TPOabs positivity as independent variable. The model was performed in three steps:

1. step 1 as crude analysis

2. step 2 as step 1 and adjusted for gender, age, and parameters, statistically (or borderline) different variables between TPOabs-positive and -negative patients, i.e. DAS28 at baseline, methotrexate (MTX) use, and IgM-RF positivity (Model 1)

3. step 3 as step 2 and adjusted for (cardiovascular) risk factors for progression in cIMT, i.e. CVD prevalence at baseline, statin use, smoking at baseline, diabetes mellitus at baseline, atherogenic index at baseline, prednisolone use at baseline, hypertension according to NCEP ATIII criteria at baseline, and TSH levels at baseline (Model 2).
Analyses were carried out using SPSS software, version 15.0 (SPSS), and $P$ values $<0.05$ were considered statistically significant.

\section{Results}

\section{Thyroid status in TPOabs-positive and -negative RA patients}

In 322 patients TPOabs status could be assessed: 47 $(14.7 \%)$ of these patients were TPOabs-positive (see Table 1). TPOabs-positive patients had significantly higher TSH levels than TPOabs-negative patients (median TSH 1.40 and $1.26 \mathrm{mU} / \mathrm{l}$ respectively, $P=0.048)$. Moreover, TPOabs levels were significantly associated with TSH $(\beta=0.01, P<0.001)$.

\section{RA characteristics in TPOabs-positive and -negative patients}

RA characteristics are shown in Table 1. The TPOabspositive group showed a significantly higher percentage of females than the TPOabs-negative group (89 vs $62 \%$; $P<0.001)$. Mean TPOabs levels were significantly lower in MTX users compared with non-MTX users. A higher percentage of TPOabs-positive was IgM-RF positive, although this did not reach statistical significance. Disease activity scores (DAS28) were higher in TPOabspositive patients compared with TPOabs-negative patients $(4.4 \pm 1.3$ vs $3.8 \pm 1.4, P=0.018)$. A significant lower percentage of the TPOabs-positive compared with TPOabs-negative RA patients was found in a low disease activity state (DAS28<3.2), respectively, 16 and 32\% (OR: 0.36, 95\% CI 014-0.88, $P=0.032)$. Adjustment for gender, MTX use, and IgM-RF slightly influenced this association (OR: 0.40, 95\% CI 0.16-1.0, $P=0.054)$.

\section{Cardiovascular risk in TPOabs-positive and -negative patients}

As shown in Table 2, no significant differences were found between TPO-positive patients and TPOnegative patients with regard to prior and baseline CVD risk factors. At baseline, cIMT was assessed in 105 randomly selected RA patients; 90 RA patients were TPOabs-negative and $15 \mathrm{RA}$ patients were TPOabs-positive.

\section{CVD risk factors in TPOabs-positive and -negative patients at follow-up}

At follow-up, 38 of the 256 (14.8\%) RA patients were TPOabs patients. At the second visit, the mean DAS28 was $3.61( \pm 1.1)$ in TPOabs-positive RA patients and $3.14( \pm 1.1)$ in TPOabs-negative RA patients $(P=0.020)$. Cardiovascular risk factors and CVD are 
Table 1 Characteristics in TPOabs-positive and -negative RA patients.

\begin{tabular}{lccc}
\hline & $\begin{array}{c}\text { TPOabs-positive } \\
n=47\end{array}$ & $\begin{array}{c}\text { TPOabs-negative } \\
n=275\end{array}$ & $\boldsymbol{P}$ value \\
\hline Demographics & & & \\
Age (years) & $62.6( \pm 7.0)$ & $63.0( \pm 7.7)$ & 0.69 \\
Female (\%) & 89 & 62 & $<0.001$ \\
Thyroid status & & & \\
TSH (mU/l) & $1.40(0.93-2.81)$ & $1.26(0.88-1.74)$ & 0.048 \\
Levothyroxine use (\%) & 8.5 & 2.1 & 0.041 \\
Manifested hypothyroid (\%) & 6.5 & 3 & 0.38 \\
Subclinical hypothyroid (\%) & 11 & 1 & 0.002 \\
RA-related baseline characteristics & & & \\
DAS28 & $4.4( \pm 1.3)$ & $3.8( \pm 1.4)$ & 0.018 \\
Low disease activity (DAS28 $<3.2)(\%)$ & 16 & 32 & 0.027 \\
ESR (mm/h) & $21(10-40)$ & $17(9-30)$ & 0.077 \\
CRP (mg/l) & $7(3-30)$ & $7(3-18)$ & 0.70 \\
RA duration (years) & $6(4-10)$ & $7(4-10)$ & 0.68 \\
Erosive (\%) & 77 & 82 & 0.41 \\
Rheumatoid factor IgM (IU/ml) & $50(9-150)$ & $31(10-142)$ & 0.40 \\
Rheumatoid factor IgM positive (\%) & 81 & 69 & 0.091 \\
ACPA (AU/l) & $30(9-450)$ & $50(10-412)$ & 0.40 \\
ACPA positive (\%) & 47 & 51 & 0.37 \\
Previous DMARDs ( $n$ ) & $2(1-3)$ & $2(2-3)$ & 0.15 \\
DMARDs current $(n)$ & $1(1-1)$ & $1(1-2)$ & 0.14 \\
MTX current (\%) & 47 & 60 & 0.094 \\
Prednisolone current (\%) & 11 & 19 & 0.23 \\
\hline
\end{tabular}

DAS28, disease activity score 28 joints; ESR, erythrocyte sedimentation rate; CRP, C-reactive protein; ACPA, anti-cyclic citrullinated protein antibody; DMARD, disease-modifying antirheumatic drug; MTX, methotrexate; $\mathrm{SSZ}$, sulphasalazine; $\mathrm{HCQ}$, hydroxychloroquine.

shown in Table 3. More TPOabs-positive patients were using lipid-lowering drugs, as $28 \%$ of the TPOabspositive RA patients compared with $13 \%$ of the TPOabsnegative RA patients were using statins $(P=0.043)$. In TPOabs-positive patients, cIMT was significantly higher compared with TPOabs-negative RA patients, 0.96 and $0.83 \mathrm{~mm}$ respectively. Linear regression analysis revealed higher cIMT progression in levothyroxine naïve TPOabs-positive patients compared with TPOabs-negative patients after (stepwise) adjustment for gender, age, disease activity at baseline, MTX use, and IgM-RF positivity ( $\beta=0.13,95 \%$ CI $0.004-0.264$, $P=0.044$, see Table 4). Additional stepwise adjustment for CVD prevalence at baseline, statin use, smoking at baseline, diabetes mellitus at baseline, atherogenic index at baseline, prednisolone use at baseline, hypertension according to NCEP ATIII criteria at baseline, and TSH levels at baseline did not influence this association $(\beta=0.13,95 \%$ CI $0.004-0.257$, $P=0.043)$.

Table 2 Baseline cardiovascular disease risk factors.

\begin{tabular}{lccc}
\hline & $\begin{array}{c}\text { TPOabs-positive } \\
n=47\end{array}$ & $\begin{array}{c}\text { TPOabs-negative } \\
n=275\end{array}$ & $\boldsymbol{P}$ value \\
\hline Cardiovascular-related factors & & & \\
Prior cardiovascular disease (\%) & 22 & 17 & 0.44 \\
CIMT $^{\mathrm{a}}(\mathrm{mm})$ & $0.82( \pm 0.15)$ & $0.81( \pm 0.12)$ & 0.72 \\
Metabolic syndrome NCEP ATIII (\%) & 27 & 22 & 0.48 \\
Smoking status & & & 0.96 \\
$\quad$ Current smoker (\%) & 29 & 30 & \\
Never smoker (\%) & 24 & 22 & \\
Former smoker (\%) & 47 & 48 & 0.93 \\
BMI (kg/m $\left.{ }^{2}\right)$ & $26.7( \pm 5)$ & $26.6( \pm 5)$ & 0.38 \\
Waist:hip ratio & $0.88( \pm 0.08)$ & $0.89( \pm 0.08)$ & 0.75 \\
Hypertension NCEP ATIII (\%) & 53 & 50 & 0.80 \\
Atherogenic index & $4.5( \pm 1.6)$ & $4.4( \pm 1.5)$ & 0.89 \\
Statin use (\%) & 9 & 11 & 0.37 \\
Glucose (mmol/l) & $5.1(4.8-5.8)$ & $5.1(4.8-5.5)$ & 0.24 \\
Hyperglycemic (>6.1 mmol/I) (\%) & 16 & 11 & 5 \\
Diabetes mellitus (\%) & 0 & 5 & \\
\hline
\end{tabular}

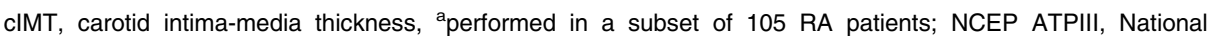
Cholesterol Education Program - Third Adult Treatment Panel. 
Table 3 Cardiovascular risk factors at follow-up in TPOabs-positive and -negative patients.

\begin{tabular}{lccc}
\hline & $\begin{array}{c}\text { TPOabs-positive } \\
n=38\end{array}$ & $\begin{array}{c}\text { TPOabs-negative } \\
n=218\end{array}$ & P value \\
\hline Cardiovascular-related factors & & & \\
Incident cardiovascular disease (\%) & 8 & 7 & 1.00 \\
CIMT $^{\mathrm{a}}$ at follow-up (mm) & $0.96( \pm 0.24)$ & $0.83( \pm 0.14)$ & $\mathbf{0 . 0 1 5}$ \\
Hypertension NCEP ATIII at follow-up (\%) & 74 & 81 & 0.38 \\
Systolic blood pressure at follow-up (mmHg) & $139( \pm 17)$ & $140( \pm 22)$ & 0.62 \\
Diastolic blood pressure at follow-up (mmHg) & $80( \pm 8)$ & $81( \pm 9)$ & 0.67 \\
Total cholesterol at follow-up (mmol) & $5.8( \pm 1.1)$ & $5.5( \pm 1.1)$ & 0.18 \\
HDL-C at follow-up (mmol) & $1.6( \pm 0.5)$ & $1.6( \pm 0.6)$ & 0.90 \\
LDL-C at follow-up (mmol) & $3.5( \pm 1.1)$ & $3.3( \pm 1.0)$ & 0.26 \\
Triglycerides at follow-up (mmol) & $1.2(1.0-1.5)$ & $1.3(1.0-1.8)$ & 0.43 \\
Atherogenic index at follow-up & $4.0( \pm 1.9)$ & $3.7( \pm 1.4)$ & 0.44 \\
Statin use at follow-up (\%) & 28 & 13 & $\mathbf{0 . 0 4 3}$ \\
Hypertriglyceridemia NCEP ATIII at follow-up (\%) & 20 & 26 & 0.53 \\
Low-HDL NCEP ATIII at follow-up (\%) & 35 & 28 & 0.44 \\
Diabetes mellitus at follow-up (\%) & 0 & 6 & 0.092 \\
BMI (kg/m ${ }^{2}$ ) & $27.5( \pm 5.1)$ & $26.8( \pm 5.0)$ & 0.49 \\
\hline
\end{tabular}

CIMT, carotid intima-media thickness, ${ }^{\text {a}}$ performed in a subset of 78 RA patients; NCEP ATPIII, National Cholesterol Education Program - Third Adult Treatment Panel.

\section{Discussion}

In this study, we found TPOabs to be present in $15 \%$ of the RA patients. Moreover, this study observed a clear association between the presence of TPOabs and thyroid status, as $\sim 18 \%$ of the TPOabs-positive patients were hypothyroid compared with only $5 \%$ of the TPOabsnegative patients. Furthermore, significant higher levels of TSH were observed in TPOabs-positive patients compared with TPOabs-negative patients. These findings are in contrast to the results of a study on 70 Italian RA patients (18), showing no association between thyroid antibody status and hormonal status. However, in a much larger study $(n=2700)$ a clear association between TPOabs and TSH levels and future hypothyroidism was seen, which underscores the observations of our study (16). In addition, our study observed a higher percentage of females in the TPOabs-positive group, which is in agreement with the results of the study by Roos et al. (16).

Interestingly, TPOabs-positive patients had a significantly higher disease activity, reflected by higher disease activity scores, a lower percentage of low disease activity and higher ESR levels in the TPOabs-positive group. An explanation for this observation might be the sex difference between TPOabs-positive and -negative patients as we observed that adjustment for gender (marginally) influenced the association between disease activity and TPO antibody status, and previous studies already demonstrated a higher disease activity state in females compared with male RA patients $(24,25)$. Moreover, lower TPOabs levels were found in the MTX users. This is in agreement with the previous observation of our group that MTX use appears to have an important immunomodulating effect on anti-glutamic acid decarboxylase in latent autoimmune diabetes of the adult and concomitant RA (26). Therefore, it is likely that the difference in disease activity between
TPOabs-positive and -negative patients is at least in part explained by a (somewhat) lower MTX use in TPOabs-positive patients. Moreover, this study observed that TPOabs-positive RA patients had higher disease activity scores during follow-up. Although these results are intriguing, the question remains whether the higher disease activity in TPOabs-positive patients is part of their phenotype and whether this results in a more therapy-refractory disease.

Moreover, this study found a larger cIMT progression, a surrogate marker for atherosclerosis in TPOabspositive patients compared with TPOabs-negative patients. Although the reported cIMT progression difference seems small, these progression rates are clinically relevant as the progression rate is tenfold bigger than the progression rate of placebo arms of large statin trials $(27,28,29)$. Previously, similar results were reported in the Rotterdam's study, as a greater incidence

Table 4 Regression coefficients of linear regression analyses in RA patients for IMT progression.

\begin{tabular}{lrr}
\hline & Model 1 & Model 2 \\
\hline TPOabs-positive & $0.134^{*}$ & $0.131^{*}$ \\
Gender & 0.042 & 0.018 \\
Age & 0.000 & -0.002 \\
DAS 28 & -0.018 & -0.022 \\
Rheumatoid factor positive & -0.022 & -0.008 \\
Methotrexate use & -0.015 & 0.008 \\
Prevalent cardiovascular disease & & $0.146^{*}$ \\
TSH & & 0.051 \\
Prednisolone use & & 0.054 \\
Statin use & & 0.033 \\
Hypertension NCEP ATIII & & -0.059 \\
Atherogenic index & & 0.015 \\
Diabetes mellitus & & 0.122 \\
Smoking & & -0.017 \\
\hline
\end{tabular}

${ }^{*} P<0.05$, NCEP ATPIII, National Cholesterol Education - Third Adult Treatment Panel. 
of aortic atherosclerosis was observed in TPOabspositive subclinical hypothyroid patients (30). These observations suggest an atherogenic role of TPOabs. In this respect, it is interesting that a previous study already suggested an atherogenic role for autoimmune thyroiditis as they observed a clear association between Hashimoto thyroiditis in an euthyroid state and cIMT independently of cardiovascular risk factors (31). Also, others observed a clear association between cIMT and hypothyroidism in female RA patients independent of cardiovascular risk factors $(32,33)$. A mechanism explaining this link may be a state of chronic inflammation in TPOabs-positive patients, which causes endothelial dysfunction, ultimately resulting in atherosclerosis. Indeed, this study observed higher inflammatory states reflected as higher disease activity scores and higher ESR rates in TPOabs-positive patients. Another explanation may be that chronic inflammation is the driving force for the cIMT progression and that TPOabs are just innocent bystanders in this case.

Although the results of this study are intriguing, some limitations have to be taken into account. The first concern is the small sample size of the study, which raises the question whether the results are generalizable to other populations. However, the small sample size might be the explanation for why no associations were observed between thyroid antibody status and (incident) cardiovascular events, as both conditions are relatively uncommon. Therefore it is difficult to reject the null hypothesis, i.e. a type II error. Obviously, a longer followup is needed to reveal the association between TPOabs status and incident CVD. Another limitation of this study is the design, as it is known that in studies with an observational design confounding is common. To deal with this multivariable regression, analyses were performed, although residual confounding of unknown and unmeasured variables cannot be excluded.

In conclusion, TPOabs positivity (irrespective of thyroid status) was associated with cIMT progression, although no increased CVD prevalence or incidence after 3 years of follow-up was observed. Moreover, this study shows TPOabs to be associated with disease activity. Hence, TPOabs seem to have a role in the amplified cardiovascular risk in hypothyroid patients.

\section{Declaration of interest}

The authors declare that there is no conflict of interest that could be perceived as prejudicing the impartiality of the research reported.

\section{Funding}

This research did not receive any specific grant from any funding agency in the public, commercial, or not-for-profit sector.

\section{Author contribution statement}

H G Raterman was responsible for the design of the study, analysis, and interpretation of data and drafting of the manuscript. A E Voskuyl,
S Simsek, B A C Dijkmans, P Lips, W F Lems, and M T Nurmohamed assisted with analysis and interpretation of data and revised the manuscript critically. M J L Peters and V P van Halm were responsible for acquisition of the data, assisted with interpretation of data, and revised the manuscript critically. I M W van Hoogstraten and M W J Schreurs performed laboratory analysis, assisted with interpretation, and revised the manuscript critically.

\section{References}

1 Dayan CM \& Daniels GH. Chronic autoimmune thyroiditis. New England Journal of Medicine 1996335 99-107. (doi:10.1056/ NEJM199607113350206)

2 Bach JF. The effect of infections on susceptibility to autoimmune and allergic diseases. New England Journal of Medicine 2002347 911-920. (doi:10.1056/NEJMra020100)

3 Anaya JM, Corena R, Castiblanco J, Rojas-Villarraga A \& Shoenfeld Y. The kaleidoscope of autoimmunity: multiple autoimmune syndromes and familial autoimmunity. Expert Review of Clinical Immunology 20073 623-635. (doi:10.1586/17446 66X.3.4.623)

4 Somers EC, Thomas SL, Smeeth L \& Hall AJ. Are individuals with an autoimmune disease at higher risk of a second autoimmune disorder? American Journal of Epidemiology 2009169 749-755. (doi:10.1093/aje/kwn408)

5 Raterman HG, van Halm V, Voskuyl AE, Simsek S, Dijkmans BA \& Nurmohamed MT. Rheumatoid arthritis is associated with a high prevalence of hypothyroidism that amplifies its cardiovascular risk. Annals of Rheumatic Disease 200867 229-232. (doi:10.1136/ard.2006.068130)

6 Lazurova I, Benhatchi K, Rovensky J, Kozakova D, Wagnerova H, Tajtakova M, Shoenfeld Y \& Macejova Z. Autoimmune thyroid disease and autoimmune rheumatic disorders: a two-sided analysis. Annals of the New York Academy of Sciences 20091173 211-216. (doi:10.1111/j.1749-6632.2009.04809.x)

7 Raterman HG, van Eijk IC, Voskuyl AE, Peters MJ, Dijkmans BA, van Halm VP, Simsek S, Lems WF \& Nurmohamed MT. The metabolic syndrome is amplified in hypothyroid rheumatoid arthritis patients: a cross-sectional study. Annals of Rheumatic Disease 201069 39-42. (doi:10.1136/ard.2008.100776)

8 Raterman HG, Nielen MM, Peters MJ, Verheij RA, Nurmohamed MT \& Schellevis FG. Coexistence of hypothyroidism with inflammatory arthritis is associated with cardiovascular disease in women. Annals of Rheumatic Disease 201271 1216-1218. (doi:10.1136/annrheumdis-2011-200836)

9 McCoy SS, Crowson CS, Gabriel SE \& Matteson EL. Hypothyroidism as a risk factor for development of cardiovascular disease in patients with rheumatoid arthritis. Journal of Rheumatology 2012 39 954-958. (doi:10.3899/jrheum.111076)

10 Effraimidis G, Strieder TG, Tijssen JG \& Wiersinga WM. Natural history of the transition from euthyroidism to overt autoimmune hypo- or hyperthyroidism: a prospective study. European Journal of Endocrinology $2011 \mathbf{1 6 4}$ 107-113. (doi:10.1530/EJE-10-0785)

11 Nielen MM, van Schaardenburg D, Reesink HW, van de Stadt RJ, van der Horst-Bruinsma IE, de Koning MH, Habibuw MR, Vandenbroucke JP \& Dijkmans BA. Specific autoantibodies precede the symptoms of rheumatoid arthritis: a study of serial measurements in blood donors. Arthritis and Rheumatism 2004 50 380-386. (doi:10.1002/art.20018)

12 De Rycke L, Peene I, Hoffman IE, Kruithof E, Union A, Meheus L, Lebeer K, Wyns B, Vincent C, Mielants H et al. Rheumatoid factor and anticitrullinated protein antibodies in rheumatoid arthritis: diagnostic value, associations with radiological progression rate, and extra-articular manifestations. Annals of Rheumatic Disease 200463 1587-1593. (doi:10.1136/ard. 2003.017574)

13 Farragher TM, Goodson NJ, Naseem H, Silman AJ, Thomson W, Symmons D \& Barton A. Association of the HLA-DRB1 gene with premature death, particularly from cardiovascular disease, 
in patients with rheumatoid arthritis and inflammatory polyarthritis. Arthritis and Rheumatism 200858 359-369. (doi:10. 1002/art.23149)

14 Strieder TG, Tijssen JG, Wenzel BE, Endert E \& Wiersinga WM. Prediction of progression to overt hypothyroidism or hyperthyroidism in female relatives of patients with autoimmune thyroid disease using the Thyroid Events Amsterdam (THEA) score. Archives of Internal Medicine $2008 \mathbf{1 6 8}$ 1657-1663. (doi:10.1001/archinte.168.15.1657)

15 Walsh JP, Bremner AP, Feddema P, Leedman PJ, Brown SJ \& O'Leary P. Thyrotropin and thyroid antibodies as predictors of hypothyroidism: a 13-year, longitudinal study of a communitybased cohort using current immunoassay techniques. Journal of Clinical Endocrinology and Metabolism 201095 1095-1104. (doi:10.1210/jc.2009-1977)

16 Roos A, Links TP, de Jong-van den Berg LT, Gans RO, Wolffenbuttel BH \& Bakker SJ. Thyroid peroxidase antibodies, levels of thyroid stimulating hormone and development of hypothyroidism in euthyroid subjects. European Journal of Internal Medicine 201021 555-559. (doi:10.1016/j.ejim.2010.09.001)

17 Wells BJ \& Hueston WJ. Are thyroid peroxidase antibodies associated with cardiovascular disease risk in patients with subclinical hypothyroidism? Clinical Endocrinology 200562 580-584. (doi:10.1111/j.1365-2265.2005.02262.x)

18 Atzeni F, Doria A, Ghirardello A, Turiel M, Batticciotto A, Carrabba M \& Sarzi-Puttini P. Anti-thyroid antibodies and thyroid dysfunction in rheumatoid arthritis: prevalence and clinical value. Autoimmunity 200841 111-115. (doi:10.1080/08916930701 620100)

19 van Halm VP, Peters MJ, Voskuyl AE, Boers M, Lems WF, Visser M, Stehouwer CD, Spijkerman AM, Dekker JM, Nijpels G et al. Rheumatoid arthritis versus diabetes as a risk factor for cardiovascular disease: a cross-sectional study, the CARRE Investigation. Annals of Rheumatic Disease 200968 1395-1400. (doi:10.1136/ard.2008.094151)

20 Arnett FC, Edworthy SM, Bloch DA, McShane DJ, Fries JF, Cooper NS, Healey LA, Kaplan SR, Liang MH, Luthra HS et al. The American Rheumatism Association 1987 revised criteria for the classification of rheumatoid arthritis. Arthritis and Rheumatism 198831 315-324. (doi:10.1002/art.1780310302)

21 Prevoo ML, van 't Hof MA, Kuper HH, van Leeuwen MA, van de Putte LB \& van Riel PL. Modified disease activity scores that include twenty-eight-joint counts. Development and validation in a prospective longitudinal study of patients with rheumatoid arthritis. Arthritis and Rheumatism 199538 44-48. (doi:10.1002/art.1780380107)

22 Expert Panel on Detection EaToHBCiA. Executive Summary of the Third Report of the Detection, Evaluation, and Treatment of High Blood Cholesterol in Adults (Adult Treatment Panel III). Journal of the American Medical Association 2001285 2486-2497. (doi:10.1001/jama.285.19.2486)

23 Van Sijl AM, Van Den Hurk K, Peters MJ, Van Halm VP, Nijpels G, Stehouwer CD, Smulders YV, Voskuyl AE, Dekker JM \& Nurmohamed MT. Different type of carotid arterial wall remodeling in rheumatoid arthritis compared with healthy subjects: a case-control study. Journal of Rheumatology 201239 2261-2266. (doi:10.3899/jrheum.120617)
24 Forslind K, Hafstrom I, Ahlmen M \& Svensson B. Sex: a major predictor of remission in early rheumatoid arthritis? Annals of Rheumatic Disease 200766 46-52. (doi:10.1136/ard.2006. 056937)

25 Iikuni N, Sato E, Hoshi M, Inoue E, Taniguchi A, Hara M, Tomatsu T, Kamatani N \& Yamanaka H. The influence of sex on patients with rheumatoid arthritis in a large observational cohort. Journal of Rheumatology 200936 508-511. (doi:10.3899/ jrheum.080724)

26 van Deutekom AW, Nurmohamed MT, Peters MJ, van Eijk IC, Dijkmans BA, Hamann D, Heine RJ \& Simsek S. Methotrexate and its effect on the anti-GAD titre in two patients with rheumatoid arthritis and diabetes mellitus. Annals of Rheumatic Disease 2008 67 1051-1052. (doi:10.1136/ard.2007.082198)

27 Furberg CD, Adams HP Jr, Applegate WB, Byington RP, Espeland MA, Hartwell T, Hunninghake DB, Lefkowitz DS, Probstfield J, Riley WA et al. Effect of lovastatin on early carotid atherosclerosis and cardiovascular events. Asymptomatic Carotid Artery Progression Study (ACAPS) Research Group. Circulation 199490 1679-1687. (doi:10.1161/01.CIR.90.4.1679)

28 Mercuri M, Bond MG, Sirtori CR, Veglia F, Crepaldi G, Feruglio FS, Descovich G, Ricci G, Rubba P, Mancini M et al. Pravastatin reduces carotid intima-media thickness progression in an asymptomatic hypercholesterolemic mediterranean population: the Carotid Atherosclerosis Italian Ultrasound Study. American Journal of Medicine 1996101 627-634. (doi:10.1016/S00029343(96)00333-6)

29 Crouse JR III, Raichlen JS, Riley WA, Evans GW, Palmer MK, O'Leary DH, Grobbee DE, Bots ML \& METEOR Study Group . Effect of rosuvastatin on progression of carotid intima-media thickness in low-risk individuals with subclinical atherosclerosis: the METEOR Trial. Journal of the American Medical Association 2007 297 1344-1353. (doi:10.1001/jama.297.12.1344)

30 Hak AE, Pols HA, Visser TJ, Drexhage HA, Hofman A \& Witteman JC. Subclinical hypothyroidism is an independent risk factor for atherosclerosis and myocardial infarction in elderly women: the Rotterdam Study. Annals of Internal Medicine 2000 132 270-278. (doi:10.7326/0003-4819-132-4-20000215000004)

31 Ciccone MM, De Pergola G, Porcelli MT, Scicchitano P, Caldarola P, Iacoviello M, Pietro G, Giorgino F \& Favale S. Increased carotid IMT in overweight and obese women affected by Hashimoto's thyroiditis: an adiposity and autoimmune linkage? BMC Cardiovascular Disorders 201010 22. (doi:10.1186/1471-2261-10-22)

32 Schott LL, Kao AH, Cunningham A, Wildman RP, Kuller LH, Sutton-Tyrrell K \& Wasko MC. Do carotid artery diameters manifest early evidence of atherosclerosis in women with rheumatoid arthritis? Journal of Women's Health 200918 21-29. (doi:10.1089/jwh.2008.0797)

33 Dessein PH, Norton GR, Woodiwiss AJ, Joffe BI \& Wolfe F. Influence of nonclassical cardiovascular risk factors on the accuracy of predicting subclinical atherosclerosis in rheumatoid arthritis. Journal of Rheumatology 200734 943-951.

Received 18 May 2013

Revised version received 29 August 2013

Accepted 4 September 2013 